LEMOS, S. et al. Osteopatia pulmonar hipertrófica associada a carcinoma broncoalveolar em um cão: relato de caso. PUBVET, Londrina, V. 6, N. 3, Ed. 190, Art. 1282, 2012.

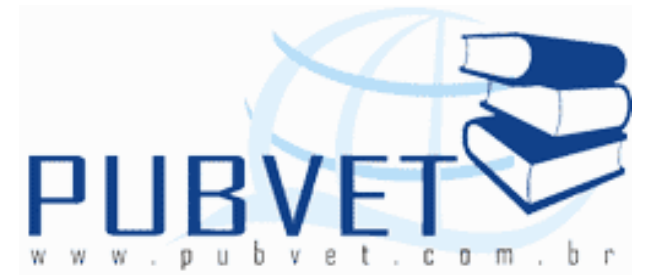

PUBVET, Publicações em Medicina Veterinária e Zootecnia.

\title{
Osteopatia pulmonar hipertrófica associada a carcinoma broncoalveolar em um cão: relato de caso
}

\section{Simone Lemos ${ }^{1}$, Juliana De Bortoli ${ }^{2}$, Mariane Pacheco dos Santos ${ }^{3}$, Paulo Henrique Zaiden Paro ${ }^{4}$, Rodrigo Supranzetti de Rezende ${ }^{4}$}

\footnotetext{
${ }^{1}$ Residente em diagnóstico por imagem no Hospital Veterinário de Uberaba ${ }^{2}$ Médica Veterinária responsável pelo Instituto Veterinário de Diagnóstico por Imagem - IVET.

${ }^{3}$ Médica Veterinária autônoma.

${ }^{4}$ Docente do curso de Medicina Veterinária, UNIUBE.
}

\section{Resumo}

A osteopatia hipertrófica pulmonar é uma doença comum em cães, tem como característica principal uma intensa reação periosteal que ocorre na região metafisária dos ossos, acomete os membros e outros ossos do esqueleto. A patogenia não é esclarecida, mas pode ser por aumento do fluxo sanguíneo para as extremidades, fatores hormonais, entre outras causas. Neoplasias pulmonares primárias são consideradas raras em cães e ocorre em cães com idade entre 10 e 11 anos e os carcinomas são os tumores mais comuns. O carcinoma broncoalveolar é um subtipo de adenocarcinoma, que se apresenta como nódulo solitário ou com padrão difuso. Este trabalho tem por objetivo relatar um caso de OHP em um cão da raça Rottwailler, macho, oito anos e 39 $\mathrm{kg}$, atendido no Hospital Veterinário de Uberaba. O animal apresentava dificuldade de locomoção, perda do apetite, aumento de volume não edematoso e indolor nos quatro membros, apatia e desidratação. Foram 
LEMOS, S. et al. Osteopatia pulmonar hipertrófica associada a carcinoma broncoalveolar em um cão: relato de caso. PUBVET, Londrina, V. 6, N. 3, Ed. 190, Art. 1282, 2012.

realizados exames laboratoriais e radiográficos, o animal foi submetido à eutanásia e o exame histopatológico confirmou a presença de carcinoma broncoalveolar.

Palavras-chave: neoplasia, pulmão, cães, osso

\title{
Hypertrophic pulmonary osteopathy associated with bronchoalveolar carcinoma in a dog: case report
}

\begin{abstract}
Hypertrophic Pulmonary Osteopathy is a common disease in dogs, whose main characteristic is an intense periosteal reaction that occurs in the metaphyseal region of bone, affecting the limbs and other bones of the skeleton. The pathogenesis is not understood, but may be by increasing blood flow to the extremities, hormonal factors, among other causes. Primary lung neoplasms are considered rare in dogs and occurs in dogs aged between 10 and 11 years and carcinomas are the most common tumors. Bronchoalveolar carcinoma is a subtype of adenocarcinoma, presenting as a solitary nodule or a diffuse pattern. This paper aims to report a case of OHP in a dog's breed Rottwailler, male, eight years and $39 \mathrm{~kg}$ The Veterinary Hospital of Uberaba. The animal had difficulty in walking, loss of appetite, swelling in non-swelling and painless in all four limbs, listlessness and dehydration. We conducted laboratory tests and X-ray, the animal was euthanized and histopathological examination confirmed the presence of a bronchoalveolar carcinoma.
\end{abstract}

Keywords: neoplasia, lung, dogs, bone

\section{Introdução:}

A osteopatia hipertrófica pulmonar (OHP) (REICHMANN et al., 2001) teve seu primeiro relato em seres humanos no final de 1800 (LENEHAN e FETTER, 1985). É considerada comum no homem e em cães, rara em outros animais (MAKUNGU et al., 2007; LENEHAN e FETTER, 1985), porém já descrita em equinos, bovinos (HEADLEY et al., 2005; BATISTA et al., 2003) e felinos (FOSTER, 2007; HEADLEY et al., 2005; BATISTA et al., 2003). Caracteriza-se 
LEMOS, S. et al. Osteopatia pulmonar hipertrófica associada a carcinoma broncoalveolar em um cão: relato de caso. PUBVET, Londrina, V. 6, N. 3, Ed. 190, Art. 1282, 2012.

por uma reação periosteal intensa nas metáfises dos ossos (ALLAN, 1998), bilateral (REICHMANN et al., 2001). No homem há acometimento das superfícies articulares, onde é conhecida como osteoartropatia hipertrófica (LIPTAK et al., 2004).

Sua patogenia não está totalmente esclarecida (GRILLO et al., 2007; HEADLEY et al., 2005; KEALY e MCALLISTER, 2005; BATISTA et al., 2003; MARTIN et al., 1971) mas acredita-se que ocorra um aumento do fluxo sanguíneo para a extremidade dos membros, junto com processos neurais mediados pelo nervo vago (GRILLO et al., 2007; HEADLEY et al., 2005; LIPTAK et al., 2004; RAHAL et al., 2003; MOREIRA et al., 2002; MARTIN et al., 1971), ou ainda fatores hormonais, hipóxicos e formação de "shunts" arteriovenosos (REICHMANN et al., 2001), que estimulam o produção excessiva de tecido conjuntivo vascular e conseqüente formação de um novo osso periosteal (MAKUNGU et al., 2007; KEALY e MCALLISTER, 2005; REICHMANN et al., 2001; ALLAN, 1998).

Os principais sinais clínicos são aumento de volume não edematoso (LENEHAN e FETTER, 1985), firme, quente, doloroso (GRILLO et al., 2007; LIPTAK et al., 2004) ou não (RAHAL et al., 2003), simétrico (RAHAL et al., 2003; LENEHAN e FETTER, 1985) e bilateral (KEALY e MCALLISTER, 2005) nas extremidades distais dos membros, além de relutância em se mover, claudicação e andar rígido (HEADLEY et al., 2005; RAHAL et al., 2003; REICHMANN et al., 2001).

Os sinais radiográficos são de reação periosteal do tipo irregular (paliçada) (ALLAN, 1998; LENEHAN e FETTER, 1985), simétricas (RAHAL et al., 2003) na região metafisária dos ossos longos (HEADLEY et al., 2005), entre eles tíbia, rádio, ulna e falanges, que tende a ficar lisa com a evolução da doença (MAKUNGU et al., 2007; KEALY e MCALLISTER, 2005; REICHMANN et al., 2001). Outros ossos afetados são a pelve (REICHMANN et al., 2001; RAHAL et al., 2003) e raramente costelas (RAHAL et al., 2003).

O carcinoma broncoalveolar é um subtipo de adenocarcinoma (MAEDA et al., 2009; RENA et al., 2003; HAYAMA et al., 2003; CARRETA et al., 2001; JUNIOR, 2000). Sua principal forma de apresentação é de nódulo solitário 
LEMOS, S. et al. Osteopatia pulmonar hipertrófica associada a carcinoma broncoalveolar em um cão: relato de caso. PUBVET, Londrina, V. 6, N. 3, Ed. 190, Art. 1282, 2012.

(MAEDA et al., 2009; RENA et al., 2003; CARRETA et al., 2001; JUNIOR, 2000; OKUBO et al., 1999; GROVER; PIANTADOSI, 1989), localizado na periferia pulmonar (EDWARDS, 1984; BARSKY, et al; 1994; RENA et al., 2003; CARRETA et al., 2001; JUNIOR, 2000; OKUBO et al., 1999), entretanto pode ser difuso e infiltrativo (CARRETA et al., 2001; JUNIOR, 2000; OKUBO et al., 1999; GROVER; PIANTADOSI, 1989), simulando pneumonia (RENA et al., 2003; JUNIOR, 2000). Descrito primeiramente em ovinos com histórico anterior de adenomatose pulmonar (EDWARDS, 1984).

Sua etiologia tem relação com doenças pulmonares anteriores que formam cicatrizes (BARSKY, et al; 1994; EDWARDS, 1984).

Os sinais clínicos evoluem de forma lenta e progressiva, pode haver pneumotórax e efusão pleural (SILVA et al., 2008; JUNIOR, 2000), entretanto, a maioria parte dos pacientes permanecem assintomáticos (OKUBO et al., 1999; GROVER; PIANTADOSI, 1989; EDWARDS, 1984). Entre os sinais podemos citar dor torácica, perda de peso, (EDWARDS, 1984) dispneia, tosse (OKUBO et al., 1999; EDWARDS, 1984).

Os principais achados radiológicos são nódulo periférico (CARRETA, et al., 2001; EDWARDS, 1984; BARSKY, et al; 1994; GROVER; PIANTADOSI, 1989; JUNIOR, 2000; OKUBO et al., 1999) com opacidade bem definida e tamanho variado (JUNIOR, 2000), entretanto pode estar difusa ou multifocal (CARRETA, et al., 2001). Deve ser feito diagnóstico diferencial para pneumonia, edema pulmonar, hemorragia e neoplasia secundária (EDWARDS, 1984). O exame radiológico do tórax pode mostrar também metástases hílares, mediastinais e atelectasia (JUNIOR, 2000). A confirmação é feita por métodos citológicos e histopatológicos (SILVA et al., 2008), entre eles lavado broncoalveolar, biópsia percutânea por agulha fina, toracotomia (RENA et al., 2003; OKUBO et al., 1999; EDWARDS, 1984).

\section{Descrição do caso:}

Foi atendido no Hospital Veterinário de Uberaba, um animal da espécie canina, da raça Rottwailler, macho, com oito anos de idade, pesando $39 \mathrm{~kg}$, 
LEMOS, S. et al. Osteopatia pulmonar hipertrófica associada a carcinoma broncoalveolar em um cão: relato de caso. PUBVET, Londrina, V. 6, N. 3, Ed. 190, Art. 1282, 2012.

com histórico de dificuldade de locomoção, perda de apetite, secreção ocular e aumento de volume dos quatro membros que segundo a proprietária havia se iniciado há um mês. Ao exame físico foi constatada apatia, desidratação leve, mucosas discretamente hipocoradas, estado nutricional ruim, aumento de linfonodos poplíteos, temperatura retal de $40,5^{\circ} \mathrm{C}$, frequência cardíaca de 122 (bpm), frequência respiratória de 32 (rpm), aumento de volume não edematoso e não doloroso em todos os membros. O animal foi submetido a exames laboratoriais complementares de hemograma, bioquímica renal e hepática, além de exames radiológicos dos membros anteriores e do tórax.

O hemograma mostrou hemácias 4,12 milhões $/ \mathrm{mm}^{3}(5,5-8,5)$, hematócrito 27,8\% (37 - 55), leucócitos totais 24600/mm (6000 - 17000). $\mathrm{Na}$ avaliação bioquímica encontrou-se as alterações de uréia 63,6 mg/dl (11 $60)$, creatinina $2,19 \mathrm{mg} / \mathrm{dl}(0,5-1,5)$, fosfatase alcalina $2835 \mathrm{U} / \mathrm{L}(0-88)$ e gama GT 17,6 U/L $(1,2-6,4)$.

O exame radiográfico dos membros anteriores do animal observou reação periosteal ativa (paliçada), simétrica em diáfise dos ossos rádio, ulna, metacarpos e falanges dos membros direito e esquerdo, não invadindo os espaços articulares (Figura 1), além de acometimento da pelve. A avaliação radiográfica do pulmão observou-se aumento de radiopacidade em campos pulmonares craniais e múltiplos nódulos radiopacos em campos pulmonares caudais, sugerindo assim a presença de neoplasia pulmonar primária e/ou metastática (Figura 2). O animal foi submetido à eutanásia, os achados macroscópicos foram de massas firmes localizadas no esterno, saco pericárdico, pulmão, fígado e linfonodo cervical, além de proliferação óssea nos em todos os membros, atingindo ossos longos e dígitos. A avaliação histopatológica dos nódulos pulmonares foi compatível com carcinoma broncoalveolar. 


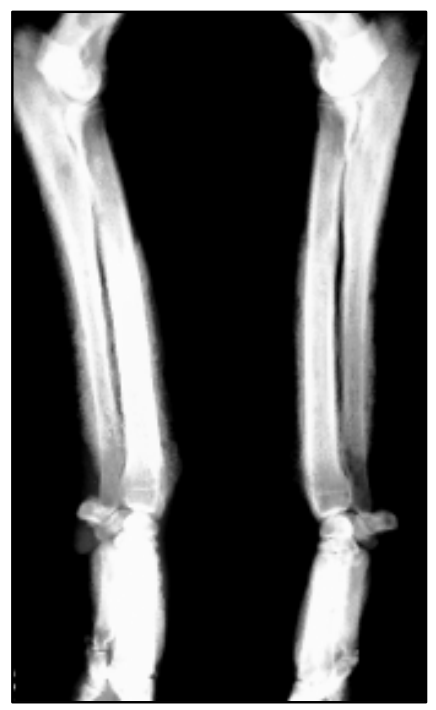

Figura1: reação periosteal ativa na metáfise de rádio, ulna e falanges.

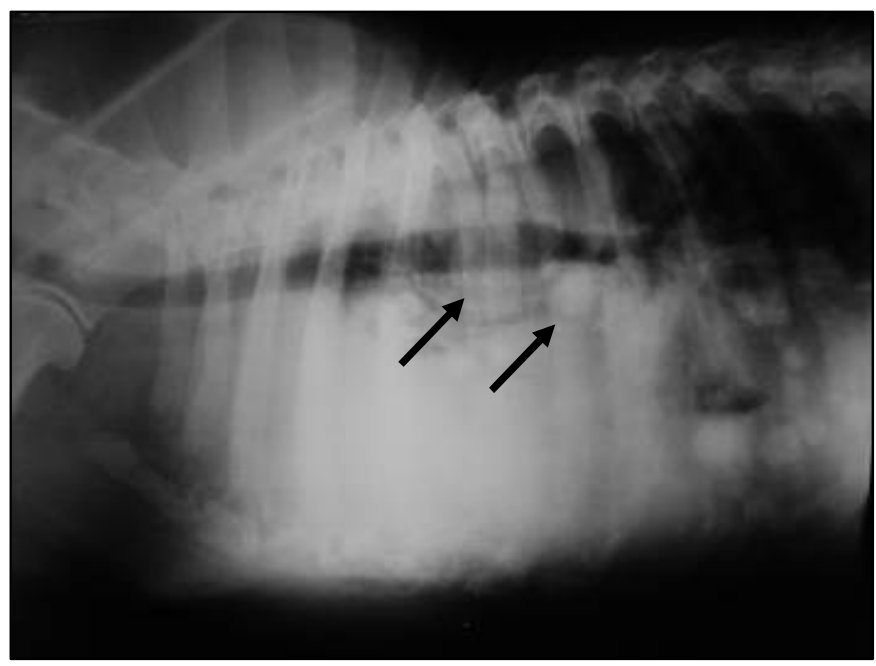

Figura2: aumento de opacidade difusa em campos pulmonares, nódulos radiopacos em lobos superiores (seta).

\section{Discussão:}

A OHP ocorre na maior parte das vezes como conseqüência secundária de processos intratorácicos neoplásicos primários, metastáticos (HEADLEY et al., 2005; LIPTAK et al., 2004; RAHAL et al., 2003) ou não (GRILLO et al., 2007; MAKUNGU et al., 2007; KEALY e MCALLISTER, 2005; RAHAL et al., 2003), como doença pulmonar inflamatória e corpo estranho intratorácico (REICHMANN et al., 2001; ALLAN, 1998). Entretanto, pode manifestar-se também como causa secundária de processos extratorácicos (GRILLO et al., 2007; LIPTAK et al., 2004; RAHAL et al., 2003) tais como neoplasias abdominais (REICHMANN et al., 2001) em órgãos como bexiga urinária, fígado e ovários (KEALY e MCALLISTER, 2005).

Os achados radiográficos encontrados nos membros anteriores são semelhantes aos citados por (MAKUNGU et al. 2007; HEADLEY et al., 2005; KEALY e MCALLISTER, 2005; RAHAL et al., 2003; REICHMANN et al., 2001; ALLAN 1998; MASEGI et al., 1994). O achado clínico de aumento de volume dos membros não edematoso corrobora com (LENEHAN e FETTER, 1985), entretanto, o mesmo encontrava-se indolor, achado esse que difere de (GRILLO et al., 2007; LIPTAK et al., 2004) e está de acordo com (RAHAL et al., 
LEMOS, S. et al. Osteopatia pulmonar hipertrófica associada a carcinoma broncoalveolar em um cão: relato de caso. PUBVET, Londrina, V. 6, N. 3, Ed. 190, Art. 1282, 2012.

2003), que relatou também a presença de hipertermia em um paciente. (MASEGI et al., 1994) relatou reação periosteal com acometimento das superfícies articulares em um guaxinim, em outros animais não há relatos de envolvimento articular.

As neoplasias pulmonares primárias em cães e gatos são raras (GUIDO et al., 2010; LUCENA, R. B, 2010), com baixa frequência (HAHN, et al., 1996), não há predisposição de raça, sexo e ocorre em animais com idade média entre 10 e 11 anos (SILVA et al., 2008). (HAHN, et al., 1996) realizaram estudos com 398 e 210 cães da raça beagle, onde apenas 8,8\% e 20\% respectivamente tiveram diagnóstico de carcinoma broncoalveolar, com idade média de 13,6 anos.

Os achados radiológicos encontrados na cavidade torácica de lesões nodulares múltiplas estão de acordo com (RENA et al., 2003; CARRETA et al., 2001; MASEGI et al., 1994. Difere de (JUNIOR, 2000; OKUBO et al., 1999) que em humanos, relataram uma única lesão radiopaca encontrada no exame radiológico na maioria dos pacientes. De acordo com (RENA et al., 2003) os lobos superiores são os mais afetados, entretanto neste estudo foram encontradas nódulos também nos lobos inferiores. Efusão pleural também foi encontrada na radiografia do tórax, já anteriormente relatada por (SILVA et al., 2008 e JUNIOR, 2000). Os sinais clínicos podem ser inespecíficos e a maioria dos pacientes são assintomáticos (OKUBO et al., 1999).

O diagnóstico definitivo é feito por avaliação histopatológica, onde se encontra células tumorais crescendo na parede e septos dos alvéolos (RENA et al., 2003; CARRETA, et al., 2001; OKUBO et al., 1999; GROVER; PIANTADOSI, 1989), sem alterar a arquitetura do interstício pulmonar (RENA et al., 2003; CARRETA, et al., 2001; GROVER; PIANTADOSI, 1989).

O tratamento é feito pela ressecção cirúrgica da neoplasia (CARRETA, et al., 2001; EDWARDS, 1984), mas pode ser realizada conforme a localização, tamanho e acometimento da cadeia linfática (EDWARDS, 1984). Características essas que determinam também o prognóstico (OKUBO et al., 1999), assim, nódulos periféricos e isolados são favoráveis (RENA et al., 2003; CARRETA, et al., 2001). Pacientes humanos com nódulo solitário tiveram sobrevida melhor 
LEMOS, S. et al. Osteopatia pulmonar hipertrófica associada a carcinoma broncoalveolar em um cão: relato de caso. PUBVET, Londrina, V. 6, N. 3, Ed. 190, Art. 1282, 2012.

em relação aqueles com lesão de caráter infiltrativo (OKUBO et al., 1999). O animal foi submetido à eutanásia devido à localização e quantidade das lesões nodulares encontradas no exame radiográfico.

\section{Referências:}

ALLAN, G. Radiographic sings of joint diasease. In: THRALL, D. E. Textbook of veterinary diagnostic radiology. 3. ed. Philadelphia: W. B. Saunders, cap. 16, p. 169-188, 1998.

BATISTA, A. A. P. ; BIANCO, J. A. P. ; BATISTA, A. P. ; ALVES, L. R. ; AFONSO, A. ; SILVA, H. S. L. ; BORGES, F. A. ; TURTELLI, C. M. ; FREIRE, M. Osteartropatia hipertrófica primária: relato de caso e revisão de literatura. Radiologia Brasileira, v. 36, n. 3, p. 183-186, 2003.

CARRETA, A.; CANNETO, B.; CALORI, G.; CERESOLI, G. L.; CAMPAGNOLI, E.; ARRIGONI, G.; VAGANI, A.; ZANNINI, P. Evaluation of radiological and pathological prognostic factors in surgically-treated patients with bronchoalveolar carcinoma. European Journal of Cardiothoracic Surgery, v.20, p. 367-371, 2001.

FOSTER, S. F. Idiopathic hypertrophic osteopathy in a cat. Journal of feline medicine and surgery, v. 9, p. 172-173, 2007.

GRILlO, T. P. ; BRANDÃO, C. V. S. ; MAMPRIM, M. J. ; JESUS, C. M. N. ; SANTOS, T. C. ; MINTO, B. W. Hypertrophic osteopathy associated with renal pélvis transitional cell carcinoma in a dog. The Canadian Veterinary Journal, v. 48, p. 745-747, 2007.

GROVER, F. L.; PIANTADOSI, S.; THE LUNG CANCER STUDY GROUP. Recurrence and Survival Following Resection of Bronchioloalveolar Carcinoma of the Lung-The Lung Cancer Study Group Experience. Ann. Surg.June 1989, vol. 209, n. 6, 1989.

GUIDO, G. W.; OliVeirA, K. P.; PEREIRA, M. F.; PAIVA, B. H. A.; CUNHA, D. F. Ocorrência de carcinoma bronquíoloalveolar e condrossarcoma em cavidade torácica em felino. Disponível em: <HTTP://www.eventosufrpe.com.br/jepex2009/cd/resumos/R0636-2.pdf>.

HAHN, F. F.; MUGGENBURG, B. A; GRIFFITH, W. C. Primary lung neoplasia in a beagle colony. Veterinary pathology, v. 33, p. 633-638, 1996.

HAYAMA, S; MORIKAWA, T; KONDOH, S; KATOH, H. Multiple early bronchioloalveolar carcinomas in both lungs. Interactive cardiovascular and thoracic surgery, v. 2, p. 506508, 2003.

HEADLEY, S. A.; RIBEIRO, E. A.; DOS SANTOS, G. J. V. G.; BETTINI, C. M.; JÚNIOR, E. M. Canine hypertrophic osteopathy associated with extra-thoracic lesions. Ciência Rural, v.35, n.4, p.941-944, 2005.

JUNIOR, E. F. P. Imaging bronchogenic carcinoma. Chest, v. 117, p. 90-95, 2000.

KEALY, J. K; McAlLiSTER, H. Ossos e articulações. In: KEALY, J. K.; McAlLiSTER, H. Radiologia e ultrassonografia do cão e do gato. 3. ed. Barueri: Manole, cap. 4, pag. 296, 2005.

LENEHAN, T. M; FETTER, A. W. Hypertrophic osteopathy. In: In: NEWTON, C. D; NUNAMAKER, D. M. Textbook of Small Animal Orthopaedics, DISPONÍVEL EM: <http://cal.vet.upenn.edu/projects/saortho/chapter 51/51mast.htm>. Acesso em: 14 jul. 2011. 
LIPTAK, J. M. ; MONNET, E. ; DERNELL, W. S. ; WITHROW, S. J. Pulmonary metastatectomy in the management of four dogs with hypertrophic osteopathy. Veterinary and Comparative Oncology, v. 2, n. 1, p. 1-12, 2004.

LUCENA, R. B; FIGHERA, R. A; CARREGARO, A. B; INKELMANN, M.A; BARROS, C. S. L. Carcinoma bronquíolo-alveolar em leão africano (Panthera leo). Pesquisa veterinária brasileira, v. 30, n. 6, p. 479-483, 2010.

MAEDA, T. Y.; CAPONE, D.; VAZ, L. C.; JANSEN, J. M. Carcinoma bronquioloalveolar. Pulmão RJ, n. 4, p. 33-38, 2009.

MAKUNGU, M.; MALAGO, J.; MUHAIRWA, A. P.; MPANDUJI, D. G.; MGASA, M. N. Hypertrophic osteopathy secondary to oesophageal foreign body in a dog - a case report. Veterinarski Arhiv, v. 77, n. 5, p. 463-467, 2007.

MARTIN, S. W.; PENNOCK, P. W.; PASS, D. Hypertrophic pulmonary osteoarthropathy in a cow. The Canadian Veterinary Journal, vol. 12, n. 6, p. 129-131, 1971.

MARTINEZ-LAVIN, M.; MANSILLA, J.; PINEDA, C.; PIJOAN, C.; OCHOA, P. Evidence of hypertrophic osteoarthropathy in human skeletal remains from pre-hispanic Mesoamerica. Annals of Internal Medicine, v. 120, p. 238-241, 1994.

MASEGI, T; YANAI, T; MATSUMOTO, C; YAMAZOE, K; NUKAYA, A; KUNIMUNE, Y; WEDA, K. Hypertrophic pulmonary osteoarthropaty in a raccoon dog (Nyctereutes procyonoides) with chronic pulmonary inflammatory lesions. Journal wildlife deseases, v. 30, n. 4, p. 612-615, 1994.

OKUBO, K.; MARK, E. J.; FLIEDER, D.; WAIN, J. C.; WRIGHT, C. D.; MONCURE, A. C.; GRILLO, H. C.; MATHISEN, D. J. Bronchoalveolar carcinoma: clinical, radiologic, and pathologic factors and survival. The Journal of Thoracic and Cardiovascular Surgery, v. 118, n. 4, p. 702709, 1999.

RAHAL, S. C.; MAMPRIM, M. J.; SEQUEIRA, J. L.; FRANCO, A. P. R. Osteopatia hipertrófica associada à metástase pulmonar de osteossarcoma em um cão. Ars Veterinária, vol. 19, n. 1, p. 035-039, 2003.

REICHMANN, P.; BARREIROS, T. R. R.; REIS, A. C. F.; OLIVEIRA, M. L. R. Osteopatia hipertrófica em equino: relato de caso. Revista Educação Continuada - CRMV.SP, v. 4, n. 2, p. $41-45,2001$.

RENA, O.; PAPALIA, E.; RUfFINI E.; CASADIO C.; FILOSSO P. L.; OLIARO A.; MAGGI G. Stage I pure bronchioloalveolar carcinoma: recurrences, survival and comparison with adenocarcinoma of the lung. European Journal of Cardio-thoracic Surgery, v. 23, p. 409414, 2003.

SILVA, M. C. V; DE NARDI; A. B; RODASKI, S. Neoplasias do sistema respiratório. In: DALECK, C. R. ; DE NARDI, A. B. ; RODASKI, S. Oncologia em cães e gatos. 1. ed. São Paulo: Rocca, 2008, cap. 21 , p. $335-344$. 